\title{
Clinical Significance of Lobar Atrophy in Frontotemporal Dementia: Application of an MRI Visual Rating Scale
}

\author{
Christopher M. Kipps ${ }^{\mathrm{a}} \quad$ R. Rhys Davies ${ }^{\mathrm{a}} \quad$ Joanna Mitchella Jillian J. Kril ${ }^{\mathrm{c}}$ \\ Glenda M. Halliday d John R. Hodges ${ }^{a}$, b \\ ${ }^{\mathrm{a}}$ Department of Clinical Neurosciences, University of Cambridge, and ${ }^{\mathrm{b}} \mathrm{MRC}$ Cognition and Brain Sciences Unit, \\ Cambridge, UK; ${ }^{\mathrm{C} D i s c i p l i n e s}$ of Medicine and Pathology, University of Sydney, and \\ dPrince of Wales Medical Research Institute, University of New South Wales, Sydney, Australia
}

\section{Key Words}

Frontotemporal dementia $\cdot$ Visual rating $\cdot$ Magnetic resonance imaging $\cdot$ Lobar atrophy $\cdot$ Behavioural variant

\begin{abstract}
Background/Aims: The status of imaging findings in the clinical diagnosis of frontotemporal dementia (FTD) remains uncertain; while they may be supportive of a diagnosis of frontotemporal dementia, they are not mandatory. Our aim was to assess patterns of lobar atrophy in a large sample of clinically defined, prospectively studied, patients using a magnetic resonance image (MRI) rating scale, to (1) determine whether imaging findings warrant a more prominent position in FTD diagnosis and (2) correlate the extent of lobar atrophy with clinical data. Methods: We adapted a recently devised post mortem rating scale for FTD to rate lobar atrophy on MRI scans. The areas rated included the frontal cortex and both anterior and posterior temporal regions bilaterally. All available brain scans from all patients seen in the Cambridge Dementia Clinic $(n=258)$ diagnosed as having FTD, together with controls $(n=20)$, were used to assess the reliability of the method. A subset of these $(n=121)$ were used for clinico-anatomic analysis. Results: The scale proved quick and reliable (intra-, inter-rater $\mathrm{k}=0.80,0.67$ ). MRI scans were abnormal in the majority of patients $(75 \%)$, with focal
\end{abstract}

atrophy present in $100 \%$ of semantic dementia (SD) patients. By contrast, nearly half (47\%) of the patients with clinical behavioural variant FTD had scans within the normal range. Behavioural cases with normal scans generally had fewer cognitive deficits and milder functional impairment than those with abnormal scans, yet displayed a clinically indistinguishable behavioural syndrome. They were not, however, simply at an earlier stage of the disease. Conclusions: MRI findings should form part of the diagnostic criteria for SD; the absence of atrophy on MRI in many behavioural cases raises the prospect that the behavioural syndrome of FTD is not specific for patients with a neurodegenerative disease.

Copyright $\odot 2007$ S. Karger AG, Basel

\section{Introduction}

Frontotemporal dementia (FTD) refers to the spectrum of non-Alzheimer dementias characterized by focal atrophy of frontal and temporal regions $[1,2]$. Three distinct clinical presentations of FTD are recognized [3]. Behavioural variant FTD (bvFTD) is characterized by progressive changes in personality including disinhibition, apathy, loss of empathy, altered eating patterns and stereotypic behaviour [4]. Two aphasic variants are also described. Progressive non-fluent aphasia (PNFA), which

\section{KARGER}

Fax +4161306 1234 E-Mail karger@karger.ch www.karger.com (c) 2007 S. Karger AG, Basel $1420-8008 / 07 / 0235-0334 \$ 23.50 / 0$

Accessible online at:

www.karger.com/dem
Prof. J.R. Hodges

MRC Cognition and Brain Sciences Unit, 15 Chaucer Road

Cambridge CB2 2EF (UK)

Tel. +44 1223355 294, ext. 862, Fax +44 1223359062

E-Mail john.hodges@mrc-cbu.cam.ac.uk 
typically results in disruption of phonological and syntactic components of language [5], and semantic dementia (SD), by contrast, in which there is progressive loss of the knowledge base underlying language, resulting in impaired comprehension with preservation of conversational fluency [6].

FTD consensus diagnostic criteria refer to frontal and/ or temporal lobar atrophy but do not mandate specific scan findings in the individual syndromes $[3,7]$. The terms 'frontal variant' and 'temporal variant' FTD, nonetheless, are widely used synonyms of bvFTD and SD, respectively. The link between temporal lobe atrophy and $\mathrm{SD}$, in particular, is well founded $[8,9]$. Furthermore, volumetric MRI studies demonstrate that atrophy may be marked even at the time of diagnosis and that this feature can distinguish between FTD and other dementing conditions [10-12]. In everyday practice, however, many cases seem to depart from the predicted patterns of atrophy.

Our aim was to devise a method for systematic assessment of structural MR images in FTD that would be quick, reliable and applicable in a wide range of settings. The main purpose of this study was (1) to investigate the relative importance of imaging findings in a large, prospectively studied, cohort of patients with a clinical diagnosis of FTD and (2) to assess the relationship of focal brain atrophy to clinical data.

\section{Method}

\section{Case Selection}

The Cambridge Dementia Clinic database was interrogated for patients carrying a diagnosis from the clinical spectrum of FTD $[3,7]$. All available clinical MR scans from these cases that included a series of coronal images were considered $(n=258)$. A further 20 scans from control subjects were added to the image series. Controls were selected from a volunteer panel of normal individuals or were the spouses of subjects under study in the Cambridge Dementia Clinic. All were comprehensively assessed (by a multi-disciplinary team including a senior neurologist, a neuropsychiatrist and in most cases a neuropsychologist) to ensure an absence of neurological or psychiatric disease (in particular, schizophrenia, mania, depression and lifelong personality dysfunction). A history (personal and family) of psychiatric or neurologic disease was sought in all cases. Cognitive screening tests were also performed [Addenbrooke's Cognitive Examination (ACE) and Mini-Mental State Examination (MMSE)]. Only a very small number of patient images $(n=2)$ were excluded because of poor image quality. The remaining 256 patient scans were placed in 2 categories based on whether the scans were performed at initial presentation or during subsequent review. In developing the method 133 review scans (plus 20 control scans) were used (see reproducibility analyses below). For clinico-anatomical analysis 125 initial scans from patients in whom a core of clinical information could be extracted from the records (FTD syndrome category; indication of overall disability within 1 year of scanning) were used (see below and results). It is important to note that the patients' diagnosis was established clinically and not on the basis of their imaging findings. While such findings were often supportive of the diagnosis, in line with the consensus criteria, scan appearance did not define the FTD subtype, and a normal scan did not exclude a diagnosis of any of the FTD subgroups. Pathological verification was available for 28 patients of the current series and in all cases upheld a diagnosis of 1 of the FTD syndromes; any individual in our database with known non-FTD pathology, regardless of prior clinical mode of presentation, was excluded from the study at inception. The research programme was approved by the Cambridge Local Research Ethics Committee.

\section{Method of Image Assessment}

We derived an MRI rating scale from a recently devised staging scheme, initially developed to rate the severity of atrophy in FTD brains post mortem that is similar to scales used in other diseases $[13,14]$. The post mortem staging has been shown to be reliable and to correlate with both disease duration and symptom severity [13]. The in vivo method, used to rate brain images in this study, involves assessment of frontal and temporal lobe atrophy at 2 defined coronal levels on MRI (see fig. 1). The process is rapid (1-2 min per case) and uses standard clinical-quality coronal MR images. In this study, scans were anonymized and their order randomized prior to rating, which was performed by 2 clinicians (R.D. and C.K.), who were blind to subject identity, diagnosis and clinical features.

The 2 coronal slices assessed are the same as those used for the post mortem rating: (I) the slice through the temporal pole just anterior to where the 'temporal stem' connects frontal and temporal lobes and (II) the slice showing the lateral geniculate nuclei (fig. 1). In all, 3 lobar regions were rated: slice I - frontal and anterior temporal; slice II - posterior temporal. A 5-point scale was adopted (e.g. rating $0=$ normal, $1=$ borderline abnormality, $4=$ most severely abnormal) with specific criteria formulated for each level (fig. 2). An array of standard reference images was used while rating to maximize consistency (fig. 2). The overall rating for each case was the highest score recorded among the lobar ratings, and we retained separate data for each lobar region. Lateralization of brain atrophy was recorded when ratings for any region were not symmetric (i.e. difference between hemispheres in any region not equal to zero).

\section{Reproducibility Analysis}

Cronbach's $\alpha$ was 0.9 for overall rating; individual lobar ratings varied between 0.8 and 0.9 , implying high internal consistency of the scale for the individual raters. Intra-rater analyses (R.D., $\mathrm{n}=60$ ) gave Cohen's $\kappa$ values for frontal, anterior temporal and posterior temporal regions of $0.82,0.83$ and 0.79 , respectively, suggesting good agreement. Inter-rater analyses (R.D. and C.K., $\mathrm{n}=256$ ) also showed substantial agreement (frontal $=0.62$, anterior temporal $=0.71$ and posterior temporal $=0.64)$. Both intra- and inter-rater reliability data compared very favourably to those of previously published MRI rating methods [11, 15]. Scores from only 1 rater (R.D.) were used in the analyses below to maximize the internal consistency of the scale. Any lobar rat- 


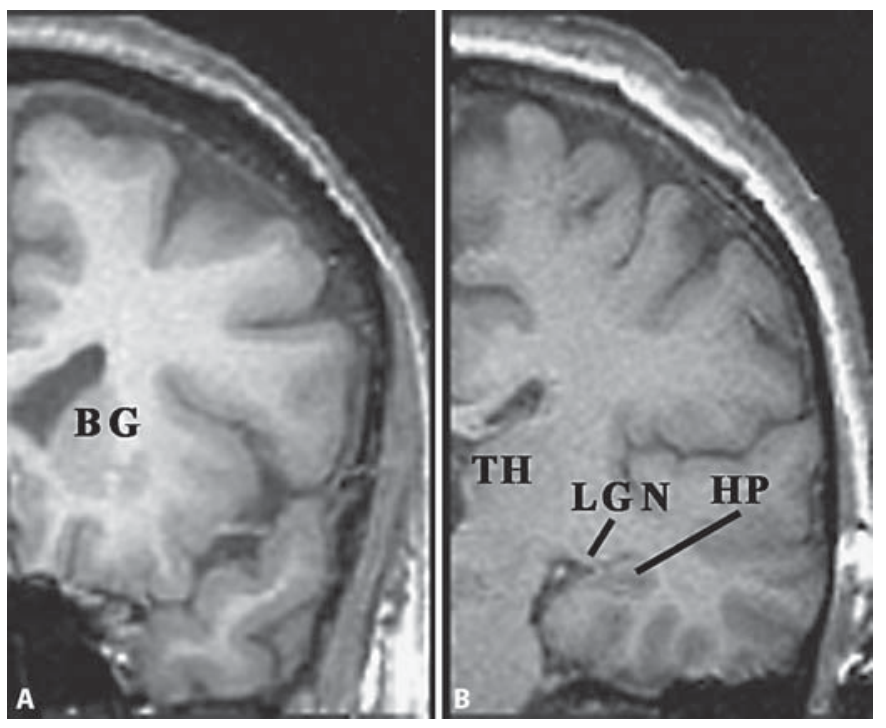

Fig. 1. Anatomical markers for rated slices. A Slice I. The most posterior scan slice through the temporal pole with no visible connection between the frontal and temporal pole. It includes frontal and temporal cortices, white matter, basal ganglia (BG) and corpus callosum. B Slice II. The slice showing the clearest view of the lateral geniculate nuclei (LGN). If contour of the nuclei is not well seen (occurs in $<10 \%$ ), the slice immediately posterior to the flattening of the medial prominence of the thalami is chosen. If LGN are equally prominent in adjacent slices, the anterior of the candidate slices is taken. It includes hippocampus (HP) in the classical 'cornu ammonis' conformation, posterior temporal cortex and white matter, posterior frontal/parietal lobes, thalami (TH; anterior pulvinar), corpus callosum and lateral ventricles (body and inferior horn).

ing that differed between the 2 raters by more than 1 point on the scale (only 17 of 1,536 individual lobar ratings) was reviewed by both raters and consensus reached on the final rating recorded.

\section{Clinico-Anatomical Analysis}

The patients were subcategorized based on the dominant clinical features into bvFTD $(\mathrm{n}=51)$, PNFA $(\mathrm{n}=22)$ and SD $(\mathrm{n}=52)$ with reference to FTD consensus criteria [3], although we retain our preferred usage of the term bvFTD to indicate the behavioural variant of FTD. Age at scanning, symptom duration to scanning and gender were documented. The Clinical Dementia Rating (CDR) was used as an indicator of overall disability [16]. Further measures of the cognitive and behavioural impairments were also available in the majority of cases. The ACE [17-19] had been performed within 1 year of scanning in 105 cases (bvFTD $=46$, $\mathrm{PNFA}=13, \mathrm{SD}=46$ ). The ACE, which has been shown to be useful in the setting of FTD [17], is a 100-point general cognitive battery, which incorporates the 30-point MMSE [20] along with further tests of language (verbal fluency, naming and comprehension), recall and visual construction. The Cambridge Behavioural Inventory $(\mathrm{CBI})$, a carer-based questionnaire covering a broad range of
Table 1. Demographic characteristics and clinical indices by FTD subtype

\begin{tabular}{lclll}
\hline & No. & $\begin{array}{l}\text { Sex } \\
\text { M/F }\end{array}$ & Age (years) & $\begin{array}{l}\text { Duration to MRI } \\
\text { (years) }\end{array}$ \\
\hline bvFTD $^{1}$ & 51 & $38 / 13$ & $60.1 \pm 7.7$ & $5.0 \pm 3.6$ \\
PNFA & 22 & $16 / 6$ & $66.3 \pm 6.8$ & $3.6 \pm 2.2$ \\
SD & 52 & $29 / 23$ & $64.4 \pm 7.1$ & $4.0 \pm 2.5$ \\
Controls & 20 & $10 / 10$ & $61.6 \pm 6.5$ & - \\
\hline All & 145 & $93 / 52$ & $62.8 \pm 7.5$ & - \\
\hline
\end{tabular}
tions.

Age and duration are expressed as means \pm standard devia-

${ }^{1}$ Age - bvFTD vs. SD, PNFA $(\mathrm{p}<0.05)$.

neuropsychiatric symptoms, validated in FTD and Alzheimer's disease [21], provided behavioural data in 77 cases $($ bvFTD $=39$, PNFA $=10, \mathrm{SD}=28$ ). Dichotomized behavioural data (abnor$\mathrm{mal}=1$, normal $=0$ ) were extracted from the CBI and summated for 13 domains (delusions, hallucinations, depressed mood, anxiety, irritability, elevated mood, agitation, apathy, sleep, disinhibition, stereotypies, dietary changes and ritualized behaviour). The latter 4 (disinhibition, stereotypies, dietary changes and ritualized behaviours) were prespecified, on the basis of previous studies, to be particularly salient features of FTD [1] and were combined to form a CBI subset score. Apathy was not included in this subset, as its discriminatory value with other neurodegenerative diseases is poor.

\section{Statistics}

SSPS 10 (SPSS Inc., Chicago, Ill., USA) was used for all statistical analyses. Comparisons were undertaken across syndrome categories (bvFTD, PNFA and SD) and rating scores. The parametric clinical data (age, symptom duration and ACE score) were compared across syndromes and rating levels by ANOVA with Scheffe post hoc tests. Gender comparisons between groups were performed using $\chi^{2}$ tests. Kruskal-Wallis tests were employed for the non-parametric data (CDR scores, total $\mathrm{CBI}$ and $\mathrm{CBI}$ subset scores, lobar rating scores and lateralization indices), with subsequent Mann-Whitney U testing as necessary. Correlational analyses for ordinal data sets were based on Spearman's analyses; in view of the multiple comparisons made, significance was taken to be $\mathrm{p}<0.01$.

\section{Results}

\section{Clinical Data}

Demographics and data are summarized in table 1. The 125 cases included 83 men and 42 women. The gender ratios did not differ significantly across syndromes. The mean age at scanning was 62.8 years ( \pm standard 


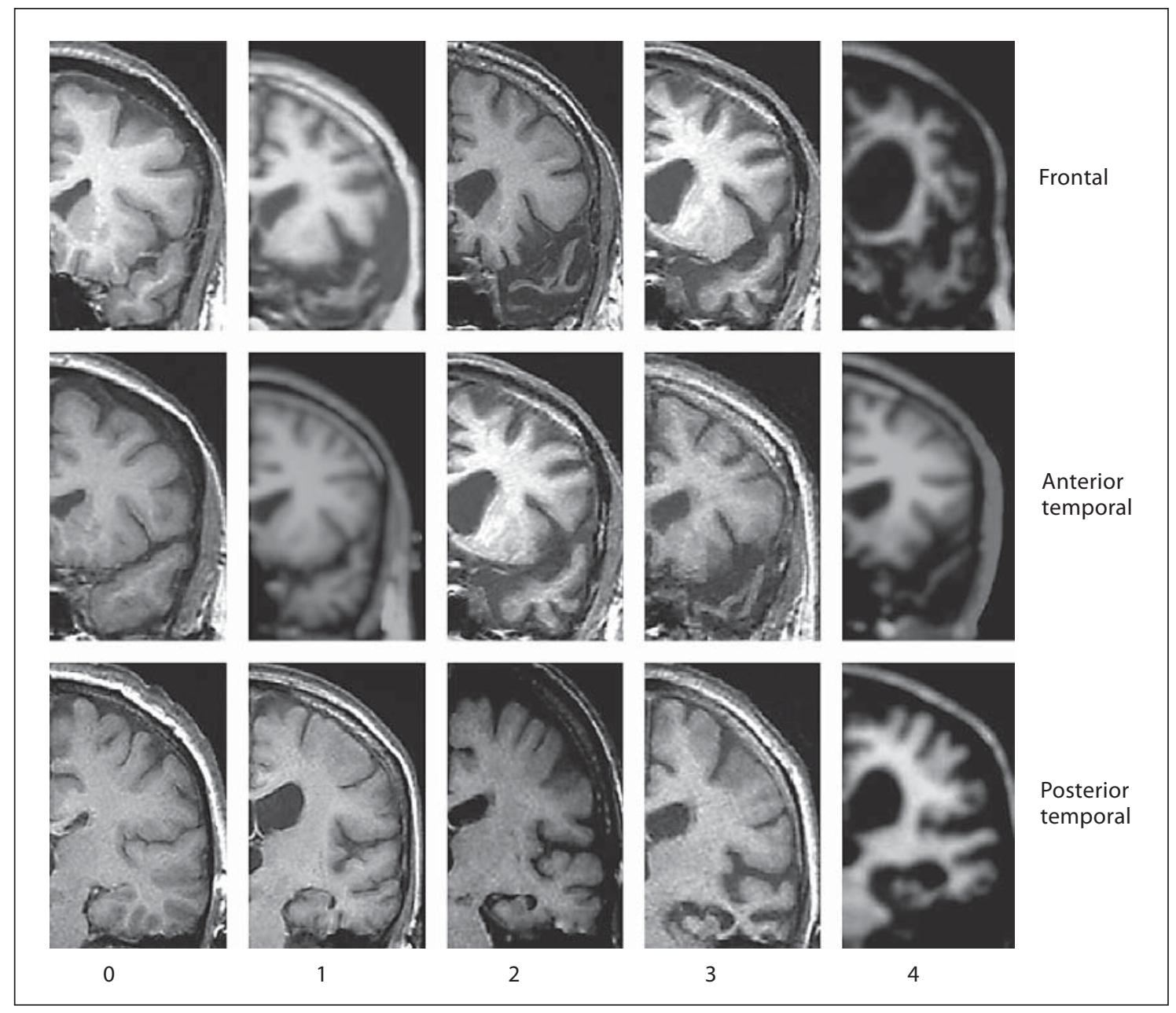

Fig. 2. Array of prerated reference images and rating criteria for lobar regions. Frontal lobe (on slice I). Stage $0=$ Normal appearances; stage $1=$ mild atrophy of orbital or supero-medial frontal cortex - contour of the basal ganglia in the lateral ventricle is convex, as in controls, but with some prominence of the lateral ventricle; stage 2 = definite sulcal widening in any cortical subregion or flattened profile to basal ganglia; stage 3 = severer cortical atrophy with clear reduction in white matter and reduced white-grey matter differentiation - stage 3 basal ganglia have concave profile; stage $4=$ cortex reduced to a ribbon and the basal ganglia virtually indiscernible. Anterior temporal lobe (on slice I). Stage $0=$ Normal appearances; stage $1=$ slight prominence of anterior temporal sulci; stage $2=$ temporal sulci definitely widened; stage 3 = gyri severely atrophic and ribbonlike - white and grey matter cannot be distinguished (normal temporal lobe at this level is less substantial than the frontal lobe, and so the ribbon-like gyri of the stage 3 temporal lobe are similar to stage 4 frontal gyri); stage $4=$ temporal pole has a simple linear profile or is not seen at all. Posterior temporal lobe (on slice II). Stage $0=$ normal appearances; stage $1=$ slight increased prominence of the lateral ventricle to form a rim around the anterior hippocampus - temporal sulci show mild prominence; stage 2 = lateral ventricle unarguably dilated with subtle reduction in hippocampal size - the medial temporal gyri may be atrophic, and there may be prominence of the temporal sulci; stage 3 = the hippocampus is small and sits at the medial tip of a greatly expanded temporal horn - sulci are definitely widened; stage 4 = hippocampus is extremely small - temporal cortex and white matter show almost complete atrophy. deviation 7.5). An ANOVA showed a significant age effect across syndromes $(\mathrm{F}=5.2$, d.f. $=3$, $\mathrm{p}<0.01)$; post hoc testing showed the bvFTD group to be significantly younger than either the PNFA $(\mathrm{p}<0.01)$ or the SD group $(p<0.05)$, but not the controls. The 2 aphasic groups did not differ significantly in age from each other or from the controls. The mean symptom duration to scanning was not significantly different between the groups $(\mathrm{F}=2.1$, d.f. $=3$, n.s.). 


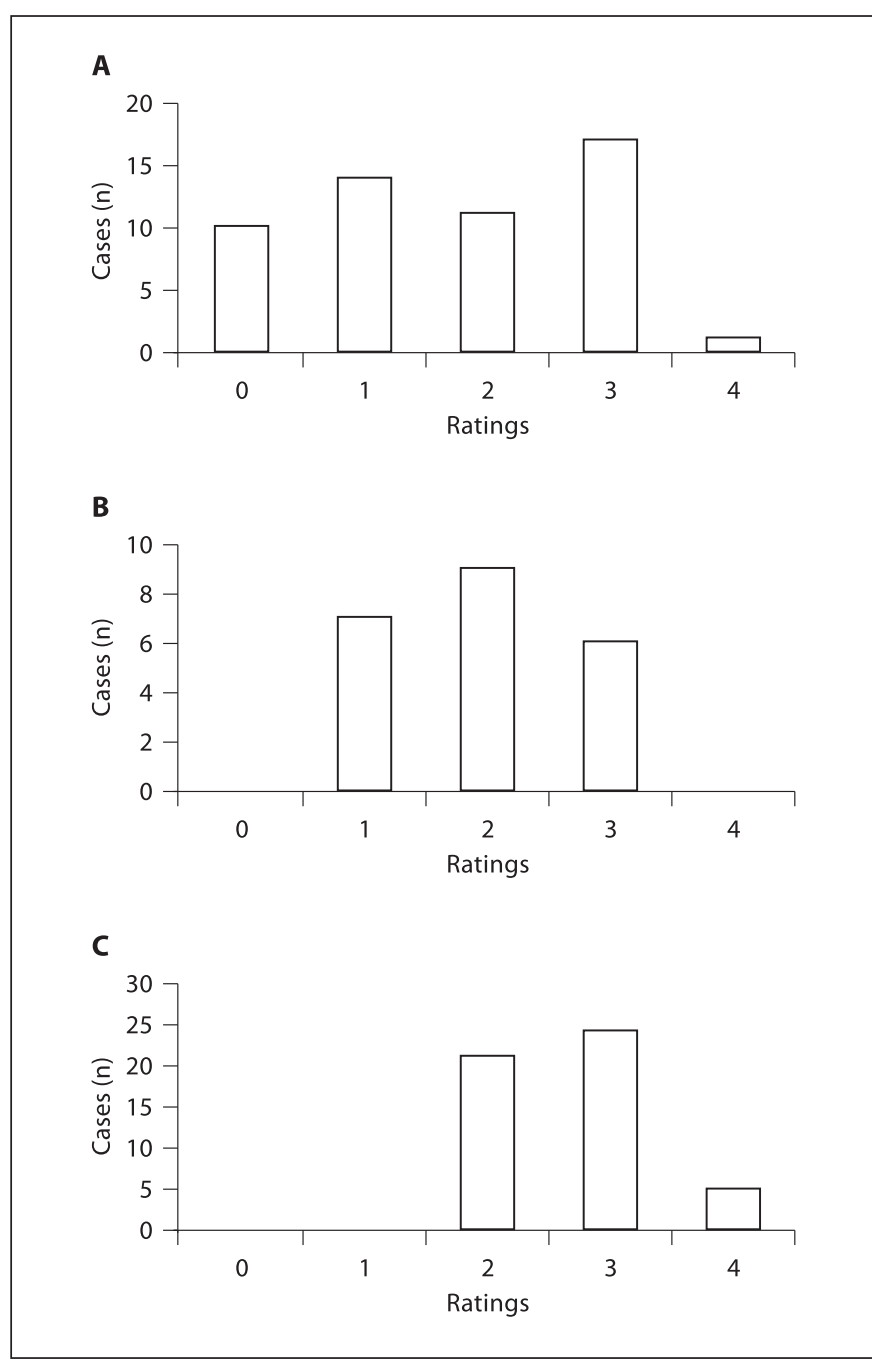

Fig. 3. Numbers of cases at each overall rating level in FTD syndromes - bvFTD (A), PNFA (B) and SD (C).

\section{Atrophy Ratings across FTD Syndromes}

Control scans were rated as 0 or 1 in all cases across all regions, thus defining the normal range of scan appearance. In the patients, a degree of cerebral atrophy (rating grades $2-4$ ) was seen in $75 \%$ of cases. All patients categorized as SD, and most with PNFA (71\%), showed clearly abnormal MRI findings, even at first scanning. By contrast, almost half (47\%) of the patients with bvFTD had a normal appearing scan (fig. 3).

Formal comparison found significant differences across syndromes for all 4 temporal subregions (anterior and posterior on the left and right) but not the frontal regions. The SD group showed significantly greater atrophy than either the bvFTD $(\mathrm{p}<0.01)$ or the PNFA $(\mathrm{p}<0.05)$

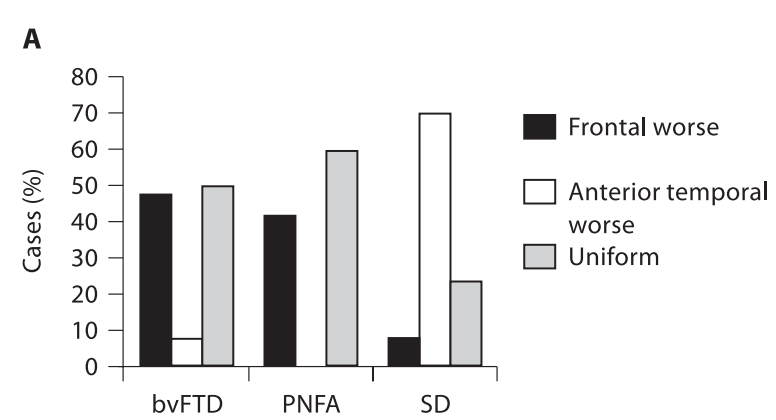

B

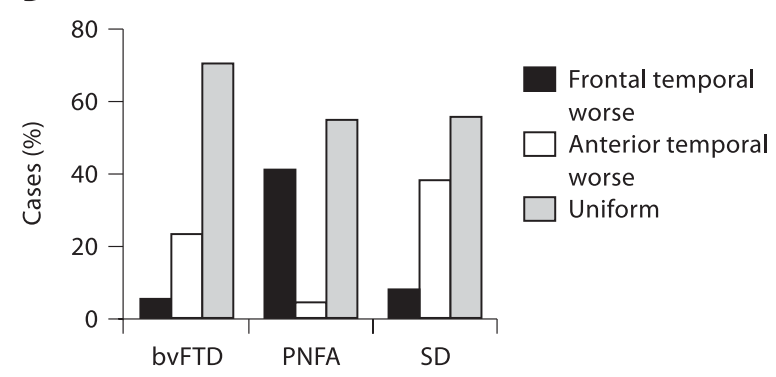

C

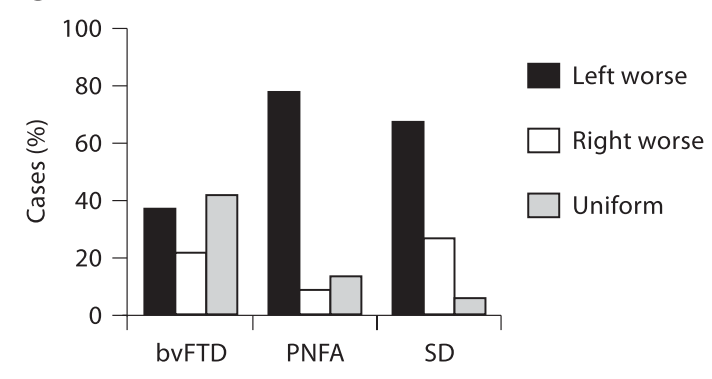

Fig. 4. Rating scores by FTD syndrome - comparison of frontal and anterior temporal (A), anterior and posterior temporal (B), and left and right ratings $(\mathbf{C})$. Note that a uniform rating can be achieved by a scan which appears normal in all lobar regions, or a scan with a similar degree of atrophy across all regions rated.

group. Comparison of the bvFTD and PNFA groups revealed greater atrophy on the left in the latter $(\mathrm{p}<0.05)$.

Relative scoring for frontal and temporal lobes in individual cases differed across syndromes, as expected (fig. 4). In bvFTD, the frontal lobe ratings were often worse than temporal ratings (49\%); the reverse was true in $67 \%$ of SD cases with a significant difference in relative frontal and temporal atrophy $(\mathrm{p}<0.001)$. Moderate numbers of both bvFTD and SD cases $(40 \%$ of the series as a whole), however, had equal frontal and anterior temporal scores. In the case of bvFTD, usually this uniformity was because all lobar regions were rated as normal. In a mi- 
Table 2. Clinical data in FTD subgroups

\begin{tabular}{lcll}
\hline & $\begin{array}{l}\text { CDR score } \\
(\max .3)\end{array}$ & $\begin{array}{l}\text { ACE score } \\
(\max .100)\end{array}$ & $\begin{array}{l}\text { CBI score } \\
(\max .13)\end{array}$ \\
\hline bvFTD & $1[0-3](39)$ & $83 \pm 10(46)$ & $8.2 \pm 3.0(39)$ \\
PNFA & $0.5[0-1](10)$ & $64 \pm 19(13)$ & $2.4 \pm 3.1(10)$ \\
SD & $0.5[0-3](28)$ & $53 \pm 30(46)$ & $5.5 \pm 3.6(28)$ \\
\hline All & $1[0-3](77)$ & $64 \pm 29(105)$ & $6.5 \pm 3.8(77)$ \\
\hline
\end{tabular}

Numbers in parentheses indicate cases in each cell. CDR scores are expressed as medians, with ranges in square brackets; $\mathrm{ACE}$ and $\mathrm{CBI}$ scores are expressed as means \pm standard deviations.

nority of cases (8\%), the atrophy profile defied the predicted gradient (i.e. temporal emphasis in bvFTD and frontal emphasis in SD). Over half the PNFA cases were rated alike for frontal and anterior temporal atrophy (59\%); the remainder had greater frontal atrophy, significantly different from SD $(\mathrm{p}<0.001)$ but not bvFTD. Anterior and posterior temporal ratings were equal in $61 \%$ of cases overall. Among the aphasic cases, there was greater anterior temporal atrophy in SD and greater posterior atrophy in PNFA $(\mathrm{p}<0.001)$.

In terms of laterality, most of the aphasic cases had left-sided atrophy (PNFA $=79 \%, \mathrm{SD}=67 \%$ ). The number of symmetrical cases of either SD or PNFA was small $(\mathrm{SD}=6 \%, \mathrm{PNFA}=14 \%)$. One quarter of the SD cases had greater right-sided atrophy. A substantial proportion of bvFTD cases had lateralized atrophy (left 37\%, right $22 \%)$. Approaching one half of bvFTD cases, however, had symmetric scan appearances, differing significantly from the PNFA $(\mathrm{p}<0.01)$ and SD $(\mathrm{p}<0.001)$ groups again this was influenced by the significant proportion of bvFTD patients with a normal scan.

\section{Clinical Data: Functional, Cognitive and Behavioural Measures}

Overall disability, indicated by CDR, differed among the syndromes $\left(\chi^{2}=20.1\right.$, d.f. $\left.=2, p<0.001\right)$, with significantly higher CDR in bvFTD than in PNFA $(\mathrm{p}<0.01)$ or SD ( $p<0.01)$; the SD group had significantly higher CDR than the PNFA cases $(\mathrm{p}<0.05)$. ACE scores also differed across syndromes $(F=22.7$, d.f. $=2, p<0.001)$, with post hoc tests confirming performance to be significantly better in the bvFTD group than either the PNFA ( $p<$ $0.01)$ or SD $(p<0.001)$ groups. The mean ACE scores for the aphasic groups were not significantly different. In

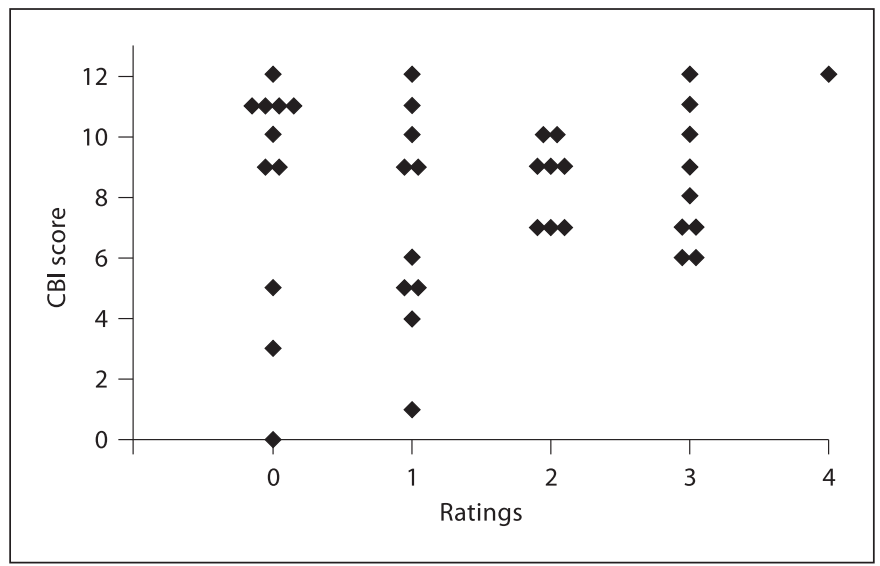

Fig. 5. CBI score plotted against atrophy rating $(n=39)$. Thirteen patients have profound behavioural disturbance (CBI of 9 or greater) but largely normal scan appearances (rated 0 or 1). One patient with a rating of 0 on the CBI was judged to have a clinical syndrome consistent with bvFTD, although this particular patient's carer only made modest endorsements on the behavioural rating scale.

parallel with the disability measure, behavioural impairment scores from the CBI showed a highly significant difference across the sample as a whole $\left(\chi^{2}=20.4\right.$, d.f. $=2$, $\mathrm{p}<0.001)$, translating into greater behavioural disturbance in the bvFTD than either the PNFA $(\mathrm{p}<0.001)$ or the SD $(\mathrm{p}<0.01)$ group. Between the aphasic groups, the $\mathrm{SD}$ cases had significantly greater behavioural disturbance $(\mathrm{p}<0.05$; table 2$)$.

\section{Relationship between Ratings and Clinical Measures}

Neither age at scanning nor symptom duration to scanning differed significantly across rating scores. The ACE scores correlated with rated atrophy (all cases/overall rating Spearman's $\rho=-0.50, p<0.001$; for each subregion $\mathrm{p}<0.05)$. The CDR scores also varied significantly with rating when all 3 groups were combined (Spearman's $\rho=-0.26, \mathrm{p}<0.01$ ).

By contrast, the summed behavioural scores extracted from the CBI did not correlate with any regional rating in the sample as a whole. Looking specifically at the bvFTD group where behavioural rather than cognitive problems dominated the clinical picture, the plot of CBI score against overall rating revealed an interesting profile with a cluster of patients showing highly abnormal behaviour but normal scan appearances (fig. 5). Correlational analysis of CBI against cases with definite atrophy ( 2,3 or 4 ratings) again failed to find a positive correlation. However, the prespecified subset of CBI scores 
deemed to be most characteristic of FTD did correlate significantly with both overall ratings (Spearmans' $\rho=$ $0.51, \mathrm{p}<0.05)$ and frontal, but not temporal, ratings bilaterally (Spearmans' $\rho$ : left frontal $=0.53$, right frontal $=$ $0.51, \mathrm{p}<0.05$ for both).

\section{Discussion}

Imaging findings enjoy an uncertain status in the diagnosis of FTD according to accepted clinical criteria [3, 7]. In this study, we describe an MRI visual rating scale for assessing brain atrophy in FTD and apply it to a large cohort of clinically defined cases. The scale is reliable, and it correlates with clinical measures of severity in the disease across the subtypes of FTD. Most importantly, application of the scale highlights a number of key relationships which have implications for clinical diagnosis and suggest a more prominent role for imaging findings in the clinical criteria for FTD.

The majority (75\%) of patients had evidence of frontal and/or temporal atrophy, which is in keeping with the published literature. Strikingly, however, nearly half (47\%) of the patients with bvFTD had a scan which was no different to the controls (rated 0 or 1 ). In contrast, all patients manifesting the clinical syndrome of SD had abnormal imaging (rated 2 or worse), as did the majority of patients with PNFA (71\%). This included many patients with scans performed at the time of clinical presentation. At least in the case of SD, these findings suggest that current consensus clinical criteria on FTD might be modified to stipulate the presence of atrophy as a requirement for diagnosis.

The profiles of lobar atrophy were in keeping with the tone of the consensus document [3]. The frontal scores were generally worse in the bvFTD cases and the temporal scores worse in SD in keeping with their respective synonyms 'frontal' and 'temporal variant'. Where bvFTD cases were classed as being 'uniform', this was invariably an artefact due to many such cases showing little or no atrophy. Over $80 \%$ of PNFA patients in this series had detectable left-sided asymmetry, even in the minority of scans which were graded as normal. Comparison of lobar atrophy between PNFA and SD showed more involvement of frontal and posterior temporal than anterior temporal regions in PNFA: frontal and posterior temporal regions are implicated in the phonological and grammatical processes affected in PNFA. These findings are in keeping with functional and structural imaging results $[22,23]$. Lateralization of atrophy was a feature of SD as well as PNFA, with the majority again showing left-sided atrophy. Even those PNFA scans that were rated as grade 1 showed a left-sided asymmetry. A significant proportion of the SD group, however, had right-sided atrophy. Such cases, said typically to display impairment in person knowledge, are well recognized [24]. By contrast, nondominant hemisphere atrophy has not been described in PNFA. Despite this, 2 PNFA cases in this series showed mainly right-sided atrophy; unfortunately, neither handedness nor other information on cerebral dominance was available.

Of the clinical measures of symptom severity, both CDR (reflecting overall functional impairment) and ACE (reflecting cognitive impairment) correlated with all 6 lobar rating points in the group as a whole. Notably, the behavioural data extracted from the CBI failed to correlate with any regional rating either in bvFTD or the aphasic groups. The absence of a correlation may be attributable to the fact that the CBI data subsume a disparate range of behavioural characteristics generated by diverse brain regions, not all of which were measured in this study. Alternatively, the range of frontotemporal scores generated by the rating scale may lack sufficient variance to adequately detect a correlation if present. The plot of CBI data and overall rating scores in bvFTD suggests an important, additional explanation. There appears to be a distinct group of patients with normal scan appearances and markedly abnormal behaviour. This impression is strengthened by the detection of a correlation between behaviour and rating scores among the bvFTD cases with abnormal scans (cases rated 0 or 1 excluded). Specifically, modest post hoc correlations were found between our prespecified behavioural measure (summed disinhibition, stereotypies, dietary changes and ritualized behaviour scores) and frontal ratings bilaterally.

How then should we view the group of bvFTD patients with normal scans? It should be noted that our normal scan bvFTD subgroup has significantly less cognitive dysfunction and less overall disability, raising the possibility that they have simply presented at an early stage of their illness. The recorded symptom duration, however, was not shorter in those with normal scanning, and the absence of a normal scan subgroup among the SD patients further suggests a different spectrum of pathologies in bvFTD. Another curiosity is that 23 out of the 24 normal scan cases were men, in contrast to the far more balanced sex ratios among bvFTD cases with abnormal scanning and among aphasic cases. We have recently described this subgroup from the bvFTD cohort in detail elsewhere [25]. In that report, patients with normal or 
borderline scans $(\mathrm{n}=15)$ showed significantly longer survival to institutionalization or death than those $(n=16)$ with definite frontotemporal atrophy $(9.3 \pm 1.7$ vs. $3.0 \pm$ 0.7 years, $\mathrm{p}<0.01)$. In the group with normal appearing scans, there was no excess of either family history or psychiatric illness. The implication is that in a subgroup of patients who meet published clinical diagnostic criteria for bvFTD but lack brain atrophy on a visual rating scale, there may be a neural basis for their symptoms distinct from the neurodegenerative pathologies generally associated with FTD.

Our main intention in this study was to assess the utility of imaging in FTD variants. It is important to highlight the fact that patients were selected on the basis of clinical and not imaging features, in line with the consensus criteria for FTD, thus avoiding problems with circular reasoning. While the imaging findings were often supportive of the diagnosis, in line with the consensus criteria, a normal scan neither excluded a diagnosis of any of the FTD variants nor defined it. The most significant consequence of this was the detection of a substantial subgroup of patients with clinically diagnosed bvFTD with normal or borderline scans, which was not a feature seen in the other clinical variants of FTD and argues against a systematic bias based on scan appearance. In addition, $8 \%$ of patients with SD or bvFTD defied the predicted gradient of atrophy (i.e. had frontal emphasis in SD and temporal emphasis in bvFTD), which further supports this contention.

The most convincing use of this scale at present would seem to be to cast significant doubt on a diagnosis of SD in the absence of significant degrees of brain atrophy, and to highlight the fact that a normal appearing scan in bvFTD suggests a much better prognosis. For cases in which there remains significant diagnostic doubt, it further provides an objective framework for longitudinal assessment and review. The use of the scale in tracking longitudinal progression of disease remains to be validated, although preliminary data (unpublished) in SD suggest that it is effective for this purpose. In this study, we have not compared imaging findings in FTD with those from other neurodegenerative diseases and therefore cannot comment on the discriminant ability of this rating scale to distinguish FTD variants from other clinical diagnoses such as Alzheimer's disease in clinical practice. Prospective validation in a wider cohort is in progress.

Our findings suggest a modification to the consensus criteria for FTD: namely that established atrophy at presentation in SD is necessary for the diagnosis. Additionally, we emphasize that a degree of caution is warranted in the diagnosis of the behavioural variant of FTD until more is known about the ultimate outcome of cases in which atrophy is absent from the MRI.

\section{Acknowledgements}

This work was supported by the Wellcome Trust (C.M.K.: grant No. 073580, and R.R.D.), the UK Medical Research Council (programme grant to J.R.H.) and the National Health and Medical Research Council of Australia (project grant to J.J.K. and G.M.H.). C.M.K. and R.R.D. are equal contributors to this work.

\section{References}

1 Hodges JR, Miller BL: Frontotemporal dementia (Pick's disease); in Hodges JR (ed): Early-Onset Dementia. Oxford, Oxford University Press, 2001, pp 284-303.

-2 Grossman M: Frontotemporal dementia: a review. J Int Neuropsychol Soc 2002;8:566583.

$>$ Neary D, Snowden JS, Gustafson L, et al: Frontotemporal lobar degeneration: a consensus on clinical diagnostic criteria. Neurology 1998;51:1546-1554.

$\checkmark 4$ Gregory CA: Frontal variant of frontotemporal dementia: a cross-sectional and longitudinal study of neuropsychiatric features. Psychol Med 1999;29:1205-1217.
5 Hodges JR, Patterson K: Nonfluent progressive aphasia and semantic dementia: a comparative neuropsychological study. J Int Neuropsychol Soc 1996;2:511-524.

-6 Hodges JR, Patterson K, Oxbury S, Funnell E: Semantic dementia: progressive fluent aphasia with temporal lobe atrophy. Brain 1992;115:1783-1806.

7 McKhann GM, Albert MS, Grossman M, Miller B, Dickson D, Trojanowski JQ: Clinical and pathological diagnosis of frontotemporal dementia: report of the Work Group on Frontotemporal Dementia and Pick's Disease. Arch Neurol 2001;58:1803-1809.

$\checkmark 8$ Galton CJ, Patterson K, Graham K, et al: Differing patterns of temporal atrophy in Alzheimer's disease and semantic dementia. Neurology 2001;57:216-225.
9 Garrard P, Hodges JR: Semantic dementia: clinical, radiological and pathological perspectives. J Neurol 2000;247:409-422.

$>10$ Laakso MP, Frisoni GB, Kononen M, et al: Hippocampus and entorhinal cortex in frontotemporal dementia and Alzheimer's disease: a morphometric MRI study. Biol Psychiatry 2000;47:1056-1063.

11 Galton CJ, Gomez-Anson B, Antoun N, et al: Temporal lobe rating scale: application to Alzheimer's disease and frontotemporal dementia. J Neurol Neurosurg Psychiatry 2001; 70:165-173.

-12 Chan D, Fox NC, Scahill RI, et al: Patterns of temporal lobe atrophy in semantic dementia and Alzheimer's disease. Ann Neurol 2001; 49:433-442. 
$\checkmark 13$ Broe M, Hodges JR, Schofield E, Shepherd CE, Kril JJ, Halliday GM: Staging disease severity in pathologically confirmed cases of frontotemporal dementia. Neurology 2003; 60:1005-1011.

14 Vonsattel JP, Myers RH, Stevens TJ, Ferrante RJ, Bird ED, Richardson EP Jr: Neuropathological classification of Huntington's disease. J Neuropathol Exp Neurol 1985;44: 559-577.

$\checkmark 15$ Wahlund LO, Julin P, Johansson SE, Scheltens P: Visual rating and volumetry of the medial temporal lobe on magnetic resonance imaging in dementia: a comparative study. J Neurol Neurosurg Psychiatry 2000;69:630635.

$\checkmark 16$ Morris JC, Edland S, Clark C, et al: The consortium to establish a registry for Alzheimer's disease (CERAD). IV. Rates of cognitive change in the longitudinal assessment of probable Alzheimer's disease. Neurology 1993;43:2457-2465.
7 Mathuranath PS, Nestor PJ, Berrios GE, Rakowicz W, Hodges JR: A brief cognitive test battery to differentiate Alzheimer's disease and frontotemporal dementia. Neurology 2000;55:1613-1620.

18 Bak TH, Rogers TT, Crawford LM, Hearn VC, Mathuranath PS, Hodges JR: Cognitive bedside assessment in atypical parkinsonian syndromes. J Neurol Neurosurg Psychiatry 2005;76:420-422.

19 Dudas RB, Berrios GE, Hodges JR: The Addenbrooke's cognitive examination (ACE) in the differential diagnosis of early dementias versus affective disorder. Am J Geriatr Psychiatry 2005;13:218-226.

20 Folstein MF, Folstein SE, McHugh PR: 'Minimental state': a practical method for grading the mental state of patients for clinicians. J Psychiatr Res 1975;12:189-198.

21 Bozeat S, Gregory CA, Ralph MA, Hodges JR: Which neuropsychiatric and behavioural features distinguish frontal and temporal variants of frontotemporal dementia from Alzheimer's disease? J Neurol Neurosurg Psychiatry 2000;69:178-186.
22 Nestor PJ, Graham NL, Fryer TD, Williams GB, Patterson K, Hodges JR: Progressive non-fluent aphasia is associated with hypometabolism centred on the left anterior insula. Brain 2003;126:2406-2418.

23 Gorno-Tempini ML, Dronkers NF, Rankin $\mathrm{KP}$, et al: Cognition and anatomy in three variants of primary progressive aphasia. Ann Neurol 2004;55:335-346.

24 Thompson SA, Graham K, Williams G, Patterson K, Kapur N, Hodges JR: Dissociating person-specific from general semantic knowledge: roles of the left and right temporal lobes. Neuropsychologia 2004;42:359370 .

25 Davies RR, Kipps CM, Mitchell J, Kril JJ, Halliday GM, Hodges JR: Progression in frontotemporal dementia: identifying a benign behavioural variant on MRI. Arch Neurol 2006;63:1627-1631. 